\title{
在 $\mathrm{Bi}(2223) / \mathrm{Ag}$ 带中产生 $I_{\mathrm{c}}$ 不可逆的原因 及其修正
}

兰玉成 郭树权 肖业皋

(中国科学院物理研究所国家超导实验室, 北京 100080)

叶斌曾荣周贻茹

(北京有色金属研究总院, 北京 100088)

\section{摘要测量了 $\mathrm{Bi}(2223) / \mathrm{Ag}$ 带材在各种强磁场取向下的超导临界电流 $\left(I_{\mathrm{c}}\right), I_{\mathrm{c}}$ 的大} 小和磁场的历史有关。这一现象可用超导体内钉扎中心的和超导颗粒之间的有效场 $\left(H_{\mathrm{eff}}\right)$ 的不可逆性来解释。 $H_{\mathrm{eff}}$ 的不可逆性来源于高温超导体内部在外磁场变化时存 在的感应持续电流和屏蔽持续电流。如果把对应于同一临界电流的两个外场 $\left(H_{\mathrm{ap}}\right)$ 的平均值, 定义为有效磁场 $\left(H_{\mathrm{eff}}\right)$, 那么 $H_{\mathrm{ap}}$ 与 $H_{\mathrm{eff}}$ 之间存在线性关系: $H_{\mathrm{eff}}=\alpha+\beta H_{\mathrm{ap}}$. $\alpha, \beta$ 值从实验数据得出后, 用 $H_{\mathrm{eff}}$ 取代 $H_{\mathrm{ap}}$, 便可得到修正的 $I_{\mathrm{c}}(H)$ 曲线.

\section{关键词不同磁场取向下 $I_{\mathrm{c}}$ 不可逆性 $I_{\mathrm{c}}$ 修正 有效场不可逆性}

高温超导体 (HTSC) 的临界电流 $I_{\mathrm{c}}$ 随外磁场的变化存在强不可逆性 ${ }^{[1 \sim n]}$. 低磁场时, 用 磁通受到挤压 ${ }^{[1]}$ 和弱联接网 ${ }^{[2]}$ 来解释, 前者已被广泛接受; 高场情况下, 也有很多物理解释, 例 如: 磁通捕获 ${ }^{[3]}$ 、磁通梯度 ${ }^{[4]}$ 、弱联接 ${ }^{[5]}$ 、有效场的不可逆性 ${ }^{[1]}$ 等, 但是都没有给出产生 $I_{\mathrm{c}}$ 不可逆 性的物理图象，也没有被广泛采用。在高场下，这还是一个有待进一步讨论解决的问题.

由于 $I_{\mathrm{c}}$ 在外磁场中有不可逆性, 所以对于相同的外磁场, 实验上存在两个 $I_{\mathrm{c}^{\prime}}$ 从从低磁场 下增加磁场 (以下简称升场) 时, $I_{\mathrm{c}}$ 较小; 从高磁场下降低磁场 (以下简称降场) 时, $I_{\mathrm{c}}$ 较大. 使 得实验测量 $I_{\mathrm{c}}(H)$ 存在较大的误差. 这两个 $I_{\mathrm{c}}$ 实验值都不能真实地反映 $I_{\mathrm{c}}$ 与磁场的关系. 为 了获得较准确的 $I_{\mathrm{c}}(H)$, 在低磁场情况下, 采用场冷方式测量样品的 $I_{\mathrm{c}}(H)$ 值, 通过对比 $J_{\mathrm{c}}(H)$ 与 $M(H)$ 曲线, 来修正 $J_{\mathrm{c}}(H)^{[12]}$.

$I_{\mathrm{c}}$ 不可逆性现象是非常复杂的, 无论从高温超导体的实际应用还是从基础研究来看都是 很重要的课题, 需要深人研究产生 $I_{\mathrm{c}}$ 不可逆的原因, 寻找修正 $I_{\mathrm{c}}$ 误差的简单易行方法. 本文 测量了在不同强磁场取向下 $\mathrm{Bi}(2223) / \mathrm{Ag}$ 带材的 $I_{\mathrm{c}}(H)$ 不可逆性; 提出了具体的物理图象, 解释 了 $I_{c}$ 不可逆性; 并提出了修正 $I_{c}(H)$ 曲线的平均方法. 


\section{1 实验}

样品由通常的 $\mathrm{Ag}$ 包套工艺制作 ${ }^{[13]}$. 把 $\mathrm{Bi}(2223)$ 超导粉末装人银管, 经过挤压、滚轧和单 轴压制, 并伴随着多次烧结而成. 样品尺寸为 $12 \mathrm{~mm} \times 2.8 \mathrm{~mm} \times 0.1 \mathrm{~mm}$. 用四引线法测量临 界电流，临界电流判据采用 $1 \mu \mathrm{V} / \mathrm{cm}$. 两电压接点相距 $5 \mathrm{~mm}$. 所有实验在 $4.2 \mathrm{~K}$ 下进行. 电 流平行于 $a-b$ 平面, 并且总是垂直于外磁场. 样品 $c$ 轴与外磁场间的夹角 $\theta$ 可从 $0^{\circ}$ 到 $180^{\circ}$ 连 续变化, 精度为 $1^{\circ}{ }^{[14]}, \theta=0^{\circ}$, 即外磁场方向与 $c$ 轴相同; $\theta=90^{\circ}$, 即外磁场平行于 $a-b$ 平面.

\section{2 结果和讨论}

图 1 为磁场的取向为 $\theta=90^{\circ}, \theta=135^{\circ}, \theta=180^{\circ}$ 时, 临界电流 $I_{\mathrm{c}}$ 与磁场的关系曲线. 从 图 1 中可看出无论外磁场取向如何, $I_{\mathrm{c}}$ 具有 很强的不可逆性, 降场时的 $I_{\mathrm{c}}$ 要比升场时的 $I_{\mathrm{c}}$ 大, 即在同一外磁场下, 由于磁场历史的 不同, 存在两个不同的 $I_{\mathrm{c}}$ 值. 另外当外磁场 $\left(H_{\mathrm{ap}}\right)$ 从高磁场下降低时, 在 $H_{\mathrm{ap}}$ 不为零处, $I_{\mathrm{c}}$ 有最大值, 而在 $H_{\mathrm{ap}}$ 为零时, $I_{\mathrm{c}}$ 反而减小，上 述现象可用样品内有效磁场 $\left(H_{\mathrm{eff}}\right)$ 的不可逆 性来解释。 $H_{\mathrm{eff}}$ 是 $H_{\mathrm{ap}}$ 和感应磁场 $\left(H_{\mathrm{id}}\right)$ 的矢 量叠加. 我们提出, 在 HTSC 中存在感应持 续电流 $\left(I_{\mathrm{ipc}}\right)$ 和屏蔽持续电流 $\left(I_{\mathrm{spc}}\right)$. $I_{\mathrm{ppc}}$ 和 $I_{\mathrm{spc}}$ 都产生 $H_{\mathrm{dd}}$. 我们首先讨论 $I_{\mathrm{ipc}}$ 和它产生的 场. 当 $H_{\text {ap }}$ 变化时 $\left(H_{\mathrm{dp}} \gg H_{\mathrm{c} 1}\right)$, 通过钉扎中 心的磁通量要随 $H_{\text {ap }}$ 的变化而变化, 并且由 此产生感应电流, 它位于钉扎中心周围的超 导区域, 是不衰减的并且环绕于钉扎中心, 用 $I_{\mathrm{ipc}}$ 表示. 在此电流环内, $I_{\mathrm{ipc}}$ 产生的感应 磁场阻止外磁场的变化, 方向与 $H_{\mathrm{ap}}$ 变化方 向相反; 但在电流环以外, 由于感应磁场是 闭合的, 其方向与 $H_{\mathrm{ap}}$ 变化方向相同. 在 HTSC 的超导颗粒内部, 一个 $I_{\mathrm{ipc}}$ 环在自 己环内产生的感应磁场被其他 $I_{\mathrm{upc}}$ 环产生的 环外感应磁场所抵消, 总的感应磁场几乎为 零. 然而, 在超导颗粒外的颗粒问, 每一个 $I_{\mathrm{ipc}}$ 环产生的感应磁场都具有相同的方向,
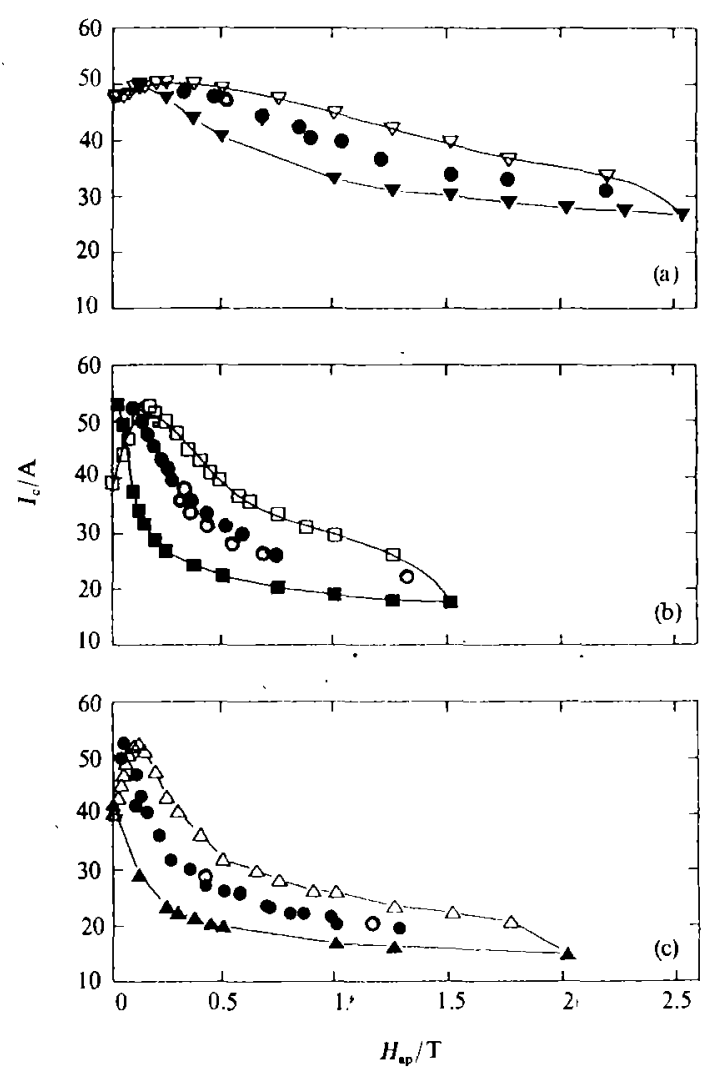

图 1 不同 $\theta$ 时的 $I_{\mathrm{c}}\left(H_{\mathrm{ap}}\right)$ 曲线 (a) $\theta=90^{\circ}$, (b) $\theta=135^{\circ}$, (c) $\theta=180^{\circ}$; 实心三角或刚方代表升场 时的 $I$ 实验值, 空心三角或四方代表降场时的 $I_{\mathrm{c}}$ 实验值，圆点 和圆圈分别为相应的 $I_{c}$ 修正值 所以在超导颗粒之间的感应磁场会很大, 可以大于 $H_{\mathrm{ap}}$, 并与 $H_{\mathrm{ap}}$ 变化方向相同. 如图 2 所 示.

下面我们讨论 $I_{\mathrm{spc}}$ ，对于传统超导体，屏蔽持续电流仅集中于表面。而对于 HTSC，由于 它具有十分强的各向异性及层状结构, $\mathrm{CuO}_{2}$ 层是超导层, $\mathrm{CuO}_{2}$ 层之间是正常层. 这样, HTSC 


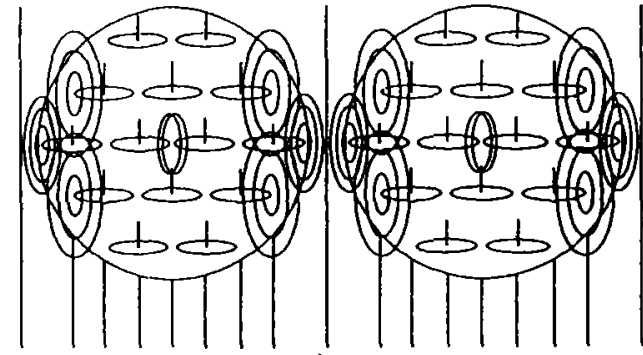

图 2 在高温超导颗粒之间的感应场 $H_{\mathrm{dd}}$ 大圆代表高温超导颗粒,直线表示通过磁通点阵的磁通线,水平 方向和垂直方向的椭圆分别表示感应持续电流 $I_{\mathrm{ipc}}$ 及其感应 场. 在 $I_{\mathrm{pc}}$ 环内感应场互相抵消 (详见正文中的讨论)，在颗粒 之间，感应场 $H_{\mathrm{id}}$ 的方向与 $H_{\mathrm{ap}}$ 变化方向相同
是超导区、弱联接区和正常区的无穷多的组 合. 当 $H_{\text {ap }}$ 发生变化时, 在超导区要产生强 $I_{\mathrm{spc}}$, 在弱联接产生弱 $I_{\mathrm{spc}}$, 在正常区内没有 $I_{\mathrm{spc}}$. 这些相邻的 $I_{\mathrm{spc}}$ 强度是如此不同, 致使它们 不能相互抵消, 结果在 HTSC 内的超导区, 仍存在较强的 $I_{\mathrm{spc}}$. 在 $I_{\mathrm{spc}}$ 环内, 它产生的场 $H_{\mathrm{id}}$ 的方向与 $H_{\mathrm{ap}}$ 变化方向相反. 在环外 (即 在超导区外的钉扎中心, 或弱联接区), $H_{\mathrm{id}}$ 的 方向与 $H_{\mathrm{ap}}$ 变化方向一致. 又因为每一个钉 扎中心周围可以存在许多 $I_{\mathrm{spc}}$ 环, 每个环产 生的 $H_{\mathrm{jd}}$ 都与 $H_{\mathrm{ap}}$ 变化方向一致. 所以在钉 扎中心处总的 $H_{\mathrm{id}}$ 是可以很大的, 可以大于 $H_{\mathrm{ap}}$. 如图 3 所示.

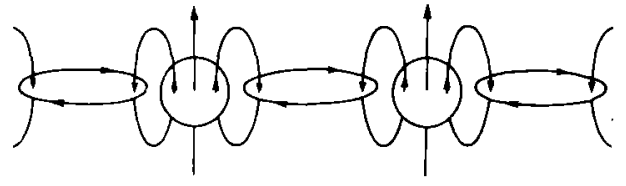

图 3 在高温超导体钉扎中心产生的感应场 $H_{\mathrm{id}}$

圆表, 小钓扎中心, 水平和垂直方向的椭圆分别表示屏蔽持续电流 $I_{\mathrm{scc}}$ 及其感应场, 直线表示磁通线, 箭头分别表示 外磁场变化方向、 $I_{\mathrm{spc}}$ 和 $H_{\mathrm{dd}}$ 的方向. 在钉扎中心,感应场 $H_{\mathrm{d}}$ 的方向与 $H_{\mathrm{ap}}$ 变化方向相同

综合上述讨论, 得到下述结论: 当 $H_{\mathrm{ap}}$ 增加时, 位于钉扎中心的和颗粒之间的 $H_{\mathrm{id}}$ (来源于 $I_{\mathrm{ipc}}, I_{\mathrm{spc}}$ ) 与 $H_{\mathrm{ap}}$ 方向一致, 造成 $H_{\mathrm{cff}}$ 大于 $H_{\mathrm{ap}}$; 又由于钉扎中心的和颗粒之间的磁场特性主章着 $I_{\mathrm{c}}$ 特性, 所以此时 $I_{\mathrm{c}}$ 实验值自然就小。相反, 当 $H_{\mathrm{ap}}$ 降低时, 在钉扎中心的和颗粒之间的 $H_{\mathrm{id}}$ 与 $H_{\mathrm{dp}}$ 方向相反, $H_{\mathrm{eff}}$ 小于 $H_{\mathrm{ap}}$, 因而测出的 $I_{\mathrm{c}}$ 较大. 且当 $H_{\mathrm{id}}=-H_{\mathrm{ap}}$ 时, $H_{\mathrm{eff}}$ 为零, $I_{\mathrm{c}}$ 出现最大 值. 当 $H_{\mathrm{ap}}$ 进一步减少时, $H_{\mathrm{eff}}$ 反号, 数值增大, 此时 $I_{\mathrm{c}}$ 值随 $H_{\mathrm{ap}}$ 的降低反而减少. $I_{\mathrm{c}}$ 的大小依 赖于外场的历史, 是由于有效场的不可逆性造成的, 这就是 $I_{\mathrm{c}}$ 产生不可逆的物理原因.

如果把同一 $I_{\mathrm{c}}$ 对应的两个磁场 (上升场、下降场) 的平均值定义为有效场 $H_{\mathrm{eff}}$, 则 $H_{\mathrm{eff}}$ 与 $H_{\mathrm{ap}}$ 应存在线性关系

$$
H_{\mathrm{eff}}=\alpha+\beta H_{\mathrm{ap}},
$$

其中, $\alpha, \beta$ 为常数. 从实验数据, 用回归法我们得到

$$
\begin{aligned}
& \text { 当 } H_{\mathrm{eff}} \text { 上升时, } \alpha=0.22, \beta=2.1, \\
& \text { 当 } H_{\mathrm{eff}} \text { 下降时, } \alpha=-0.075, \beta=0.66 \text {, }
\end{aligned}
$$

从 (1), (3) 式我们能看出, 在降场时当 $H_{\mathrm{ap}}=-\alpha / \beta$ 时, $H_{\mathrm{eff}}$ 为零, 此处 $I_{\mathrm{c}}$ 应有极大值 $\left(H_{\mathrm{ap}}=0\right.$ 时, $H_{\mathrm{eff}}$ 不为零, $I_{\mathrm{c}}$ 反而较小). 从(1) 式计算出 $H_{\mathrm{eff}}$ 值后, 用有效场 $H_{\mathrm{eff}}$ 取代外磁场 $H_{\mathrm{ap}}$, 则可修 正 $I_{\mathrm{c}}$ 值; 修正后的 $I_{\mathrm{c}}\left(H_{\mathrm{eff}}\right)$ 曲线绘于图 1. 实心圆为升场时 $I_{\mathrm{c}}$ 的修正值, 空心圆为降场时 $I_{\mathrm{c}}$ 的修正值. 修正后的 $I_{\mathrm{c}}\left(H_{\mathrm{eff}}\right)$ 曲线消除了不可逆现象. $I_{\mathrm{c}}\left(H_{\mathrm{eff}}\right)$ 曲线不依赖于磁场历史, 可以较 真实地反映临界电流与磁场的关系. 


\section{3 结论}

我们测量了 $\mathrm{Bi}(2223) / \mathrm{Ag}$ 带材在不同磁场取向条件下的 $I_{\mathrm{c}}(H, \theta)$ 不可逆性. 我们提出, 在 强场下, HTSC 中存在感应持续电流和屏蔽持续电流, 它们产生的感应磁场在钉扎中心及在超 导颗粒之间不仅是不可逆的, 而且可以是很大的, 主宰了 $I_{c}(H, \theta)$ 不可逆性. 我们认为在颗粒 超导体中, 以及其他内部存在屏蔽超导电流的超导体中, 都会产生临界电流的不可逆性。由 于 $I_{\mathrm{c}}$ 不可逆性会引起很大的 $I_{\mathrm{c}}$ 实验误差, 我们提出用有效磁场替代外加磁场来修正, 用此方 法可以获得与外磁场历史无关的、真正反映 $I_{\mathrm{c}}(H)$ 关系的超导临界电流.

\section{参考 文 献}

1 Evetts J E, Glowacki B A. Relation of critical current irreversiblity to trapped flux and microstructure in polycrystalline $\mathrm{YBa}_{2} \mathrm{Cu}_{3} \mathrm{O}_{7}$. Cryogenics, 1988, 28: 641 649

2 Chen K Y, Qian Y J. Critical current and magnetoresistance hysteresis in polycrystalline $\mathrm{YBa}_{2} \mathrm{Cu}_{3} \mathrm{O}_{7_{-x}}$. Physica $\mathrm{C}$, 1989, $159: 131 \sim 136$

3 Kwasnitza K, Widmer Ch. Hysteretic effects in the flux-flow state of granular high- $T_{\mathrm{c}}$ superconductors. Physica C, $1990,171: 211 \sim 215$

4 Majoros M, Polak M, Strbik V et al. Hysteresis of transport critical currents in high-temperature $\mathrm{YBa}_{2} \mathrm{Cu}_{3} \mathrm{O}_{7}$ superconductors: bulk samples and thin films. Supercond Sci Technol, 1990, 3:227 232

5 Watanabe $\mathrm{K}$, Noto $\mathrm{K}$, Morita $\mathrm{H}$ et al. Anisotrpy and hysteresis of transport critical currents in high temperature Ln-Y-Ba$\mathrm{Cu}-\mathrm{O}$ superoonductors. Cryogenics, 1989, 29:263 267

6 Huang W F, Hung K J, Shen J G. Mangetic hysteresis of transport critical current in polycrystalline YBCCO. Physica C, 1993, 208: 7 10

7 Altshuler E, Garcia S, Barroso J. Flux trapping in transport measurments of $\mathrm{YBa}_{2} \mathrm{Cu}_{3} \mathrm{O}_{7-x}$ superconductors. Physcia $\mathrm{C}$, 1991, 177: $61 \sim 66$

8 Mattews D N, Rusell G J, Taylor K N R. Flux trapping energies in $\mathrm{YBa}_{2} \mathrm{Cu}_{3} \mathrm{O}_{7-x}$ in the presence of a transport current. Physica C, 1990, 171:301 304

9 Ueyama M, Hikata T. Kato T et al. Microstructures and $J_{c}$-B characteristics of Ag-sheathed Bi-based superconducting wires. Japanese Journal of Applied Physics, 1991, 30(8A): L3 184 L1 386.

10 Osanura K, Oh S S, Ochiai S. Effect of thermomechanical treatment on the critical current density of Ag-sheathed B(Pb)SCCO tapes. Supercond Sic Technol, 1990, 3(3): 143 147

11 Guo Shuquan, Lan Yucheng, Ye Bin et al. The cricitical current irreversibility and modification of Bi(2223)/Ag tapes at high fields. Physica C, 1994, 235-240: $3025 \sim 3026$

12 曾朝阳, 史可信, 丁世英等。篻化物超导体低场临界电流不可逆性和可能的 $J_{c}(H)$ 曲线修正. 低温物理学报, 1993, 15(2): $110 \sim 114$

13 Zhou Yiru, Ye Bin, Zhang Jianguo et al. Preparation, microstructure and properties of Ag-heathed Bi(Pb)Sr-Ca-Cu-O superoonductor tapes. Rare Metals, 1992, 11(2):120 124

14 Guo Shuquan, Qin Xiaomei, Zhang Jinglong et al. Angular dependence of critical curent in Ag-sheathed Bi(2223) superconductor tape. Chinese Phys Lett, 1993, 10(9): 566 568 